American Journal of Nanotechnology 1 (2): 45-50, 2010

ISSN 1949-0216

(C) 2010 Science Publications

\title{
The Ultra-Precision Polishing of Large Aperture Reaction Bonded Silicon Carbide Mirror
}

\author{
Yong Shu, Yifan Dai, Ziwen Zheng and Shengyi Li \\ College of Mechatronic Engineering and Automation, \\ National University of Defense Technology, Changsha 410073, China
}

\begin{abstract}
Problem statement: Silicon Carbide ( $\mathrm{SiC}$ ) optical materials have become the first choice for mirrors in space optical systems and large ground-based optical systems due to their outstanding mechanical, physical and optical properties. Compared with traditional optical glasses, SiC optical materials are provided with the characteristics of high hardness and multiphase, which embarrass the high efficient fabrication of SiC mirrors with ultra-smooth surfaces and high precision. Approach: We choose some typical polishing parameters and conduct a series of experiments, trying to find out the relationship between polishing parameters and resulting surface roughness. The polishing parameters for Reaction Bonded Silicon Carbide (RB-SiC) are optimized from the analysis of these experiments. Then we can apply these parameters to the figuring process. Computer Controlled Optical Surfacing (CCOS) is a widely used deterministic polishing method and features in low-cost, high-precision and large flexibility. The basic theory and process of CCOS are given in detail. Then we employ CCOS in the ultra-precision machining of RB mirrors. Results: We polish a RB SiC sample with the optimized polishing parameters and a surface roughness better than $1 \mathrm{~nm}$ (RMS) is obtained. A $475 \mathrm{~mm}$ diameter sphere RB-SiC mirror with a relative aperture of $1: 1$ is polished by CCOS and the surface error reduces to $0.175 \lambda(\mathrm{PV}) / 0.009 \lambda(\mathrm{RMS})$ from $0.526 \lambda(\mathrm{PV}) / 0.080 \lambda(\mathrm{RMS})$. Both results represent a very smooth surface and a very precise figure. Conclusion: The result proves the feasibility of the polishing technology and CCOS method. These works also accumulate experience for the manufacturing of aspheric $\mathrm{SiC}$ mirror.
\end{abstract}

Key words: Reaction bonded, silicon carbide, RB-SiC mirror, ultra-smooth polish, Computer Controlled Optical Surfacing (CCOS), surface error, optical systems, polishing parameters, optical materials, large aperture, traditional materials

\section{INTRODUCTION}

Along with the development of space optical systems and large ground-based optical systems such as large-scale telescope, early-warning satellite, detecting satellite, meteorological satellite, super laser and laser radar, the diameters of mirror in these optical systems become a key factor that should be meet. As the diameter of mirror increases, the sensitometer will collect more light, which means we can get a better graph. But the system will be much heavy as the diameter of mirror increases. The additional weight will lead to deformation which challenges the precision of mirror and also result in a rising cost of launching it to orbit. Therefore, we need to figure out some methods to solve these mentioned problems. One feasible plan is to optimize the structure while the other way is to employ new material with more excellent properties. For space optical system, mirror will face a more rigorous environment such as ultra-low temperature, stronger radiation and so on. So the material used for these mirrors should have following properties such as relative low density, high specific stiffness, great heat conductivity, appropriate strength and low hardness.

$\mathrm{SiC}$ optical material has become a very promising material for large reflector due to its outstanding properties. Compared to traditional materials, $\mathrm{SiC}$ has excellent physical properties such as high Young's modulus, moderate density, small expansion coefficient, strong resistance of thermal shock, high specific stiffness and high stability of dimension (Peter and Krodel, 2010; Sus et al., 2010). The RB SiC has an advantage over other kinds of $\mathrm{SiC}$ that it can be fabricated in nearly any kinds of configurations. However, it's a great challenge to get a low surface roughness due to the different hardness of $\mathrm{SiC}$ and $\mathrm{Si}$ components in $\mathrm{RB} \mathrm{SiC}$. Generally speaking, polishing $\mathrm{RB} \mathrm{SiC}$ optical material with pitch and diamond polishing powder (Kang et al., 2008) can get good surface roughness.

Corresponding Author: Yong Shu, College of Mechatronic Engineering and Automation,

National University of Defense Technology, Changsha 410073, China 
After obtaining a good surface, we should focus on how to get a good figure. The Computer Controlled Optical Surfacing (CCOS) (Zheng et al., 2010; Arneson et al., 2010) uses a small, rotating tool which travels over the surface of workpiece with specified tool-path and velocity under the control of computer. By accurately control of parameters like resident time or polishing pressure, a prescribed amount of material can be removed at each point. Nowadays, CCOS has been widely used in the manufacturing of mid and large aperture, high accuracy and steep optical components. Our goal is to employ CCOS for the high precision fabrication of $\mathrm{RB} \mathrm{SiC}$ mirrors.

\section{MATERIALS AND METHODS}

In this section, we mainly introduce the methods to fabricate a high quality $\mathrm{RB} \mathrm{SiC}$ mirror. We face two difficulties: first is how to get an ultra-smooth surface while another is how to obtain a high precision figure.

Polishing of RB SiC: In order to get an ultra-smooth surface, we can polish $\mathrm{RB} \mathrm{SiC}$ with diamond and pitch as we mentioned before. We need to find out which kind of diamond and pitch we should apply to get best result during the polishing process. While at the same time, there are other factors which are very import to the resulting surface roughness, like the polishing time and how to combine different kinds of diamond and polishing pad together to get best surface quality with proper efficient.

Before the polishing experiment, we should learn something about $\mathrm{RB} \mathrm{SiC}$. The preparation of Reaction Bonded Silicon Carbide (RB-SiC) (Zhang et al., 2010) starts with press molding of the slurry containing alpha silicon carbide, carbon, some additive and water. A cellular biscuit will be obtained after drying. The biscuit is sintered in liquid silicon at a temperature significantly higher than the melting point of silicon. These procedures produce a near dense silicon carbide body. Typically, the body contains $79 \%$ of alpha $\mathrm{SiC}$, $17 \%$ of beta $\mathrm{SiC}$ and $3 \%$ of $\mathrm{Si}$. The different hardness of $\mathrm{SiC}$ and $\mathrm{Si}$ embarrass the ultra smooth polishing of $\mathrm{RB} \mathrm{SiC}$. Based on our existing experience of polishing $\mathrm{SiC}$ material, experiments are conducted under the conditions listing in Table 1 to get the rules between polishing parameters and resulting surface roughness.

We make up our own polishing slurry. The slurry contains water, diamond powder and some additive. The mass concentrations of all kinds of slurries are $1 \%$. After put all materials together, we stir the slurry while vibrating it in an ultrasonic vibration machine for half an hour. Then we get uniformly distributed suspending slurry.
Experiments are conducted on several $60 \mathrm{~mm}$ diameter pre-polished circle RB-SiC samples which were cut from a bigger material and each sample was polished under the condition listed in Table 1. Ten equispaced points along the diameter of the polished sample are tested by Zygo New View 200 and the given result is averaged from these ten points.

Basic theory of CCOS: In order to get a high precision figure error, we need to employ CCOS for the figure improving. First we should know some basic theory of CCOS.

During CCOS process, a polishing pad moves on a workpiece and is held in contact with the workpiece by a constant force. The material removal at any point on the workpiece depends upon the pressure $\mathrm{P}$ and the relative velocity $\mathrm{V}$ between polishing pad and workpiece at that point. According to Preston Equation (Leal-Cabrera and Cordero-Davila, 2009), the removal is given by:

$\frac{\mathrm{dz}}{\mathrm{dt}}=\mathrm{KPV}$

where, $\mathrm{K}$ is a constant of proportionality that depends upon all the factors that affects polishing process except pressure and relative velocity.

Removal function (Chen et al., 2010) is defined as the average material removal amount of polishing pad during unit time without shifting and is given by:

$R(x, y)=\lim _{T \rightarrow \infty}\left(\frac{1}{T} \int_{0}^{T} \Delta Z_{r}(x, y, t) d t\right)$

where, $\mathrm{R}(\mathrm{x}, \mathrm{y})$ is removal function, $\Delta \mathrm{Zr}(\mathrm{x}, \mathrm{y}, \mathrm{t})$ is material removal amount without shifting the pad. Material removal of the whole workpiece can be got by shifting removal function along the workpiece and accumulating material removal at every area.

Assuming that profile and surface error of workpiece won't affect removal function and polishing process. Based on additivity of material removal and linearity between removal and time, the relationship of material removal amount, removal function and resident time can be written as:

$\Delta \mathrm{Z}(\mathrm{x}, \mathrm{y})=\mathrm{R}(\mathrm{x}, \mathrm{y}) * * \mathrm{~T}(\mathrm{x}, \mathrm{y})$

where, ** means two-dimensional convolution. From measured surface error $\Delta \mathrm{Z}(\mathrm{x}, \mathrm{y})$ and removal function $\mathrm{R}(\mathrm{x}, \mathrm{y})$, we can calculate resident time $\mathrm{T}(\mathrm{x}, \mathrm{y})$. The surface error could be eliminated by polishing workpiece under the control of $\mathrm{T}(\mathrm{x}, \mathrm{y})$. 
Am. J. Nanotech., 1 (2): 45-50, 2010

Table 1: Parameters of RB-SiC polishing experiments

\begin{tabular}{|c|c|c|c|c|c|c|}
\hline \multirow[b]{2}{*}{ Serial number } & \multirow[b]{2}{*}{ Basic process parameters } & \multirow[b]{2}{*}{ Research variables } & \multicolumn{4}{|c|}{ Values of variables } \\
\hline & & & 1 & 2 & 3 & 4 \\
\hline Prepare & MPP: 55\# pitch PP: $10 \mathrm{kPa}$ VPD: $45 \mathrm{rpm}$ & & & & & \\
\hline Polishing & SPG: W0.5 & --- & --- & --- & --- & --- \\
\hline 1 & MPP: KSP 66A PP: $10 \mathrm{kPa} \quad$ VPD: $45 \mathrm{rpm}$ & SPG $(\mu \mathrm{m})$ & 0.5 & 2.5 & 7 & --- \\
\hline 2 & MPP: KSP 66A SPG: W0.5 VPD: $45 \mathrm{rpm}$ & $\mathrm{PP}(\mathrm{kPa})$ & 5 & 10 & 15 & 20 \\
\hline 3 & MPP: KSP 66A～SPG: W0.5 PP: $10 \mathrm{kPa}$ & VPD(rpm) & 30 & 40 & 50 & 60 \\
\hline 4 & PP: $10 \mathrm{kPa} \quad$ SPG: W0.5 VPD: $45 \mathrm{rpm}$ & MPP & KSP 66A & 64\# pitch & 60\# pitch & 55\# pitch \\
\hline
\end{tabular}

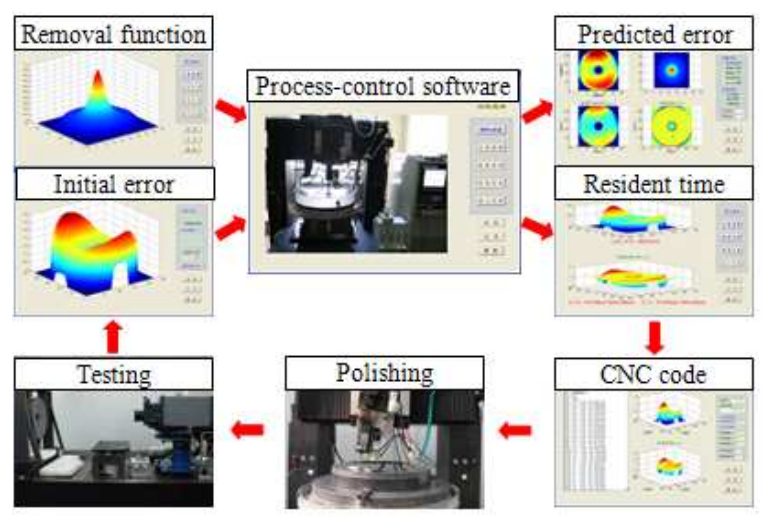

Fig. 1: CCOS flow diagram

CCOS process: The CCOS process uses an iterative method which is showed in Fig. 1 to polish the workpiece. The surface is measured by interferometry or profilometry. If measured surface error reaches to desired requirement, the process is over, or another polishing process is necessary. Key CCOS parameters are determined by existing surface error.

Surface error is analyzed by computer and the result is input into process-control software together with removal function. The software calculated resident time and predicted residual error after polishing. If the predicted result is unacceptable, the parameters are changed and the step repeated. Once an acceptable result is predicted, a CNC code file is generated and inputted into CCOS machine. The workpiece is polished with the prescribed parameters by CCOS machine under the control of CNC. The entire cycle is repeated to achieve the desired figure goal.

A $475 \mathrm{~mm}$ diameter sphere $\mathrm{RB} \mathrm{SiC}$ mirror was polished on our Aspheric Optical Compound Machining Tool (AOCMT). To obtain a good surface roughness, we applied pitch polishing pad, proper diamond polishing powder, relatively low polishing pressure and polishing velocity to polishing process. The pitch polishing pad was changed every $1.5 \mathrm{~h}$ to keep it in good condition.
A Zygo GPI XP/D interferometer was employed to measure the workpiece. After one CCOS cycle, the mirror was moved to a testing room and supported by a belt. In order to eliminate distortion of the belt and the mirror, the mirror would be held still on the belt for at least 8 hours before testing. Special attention should be given to the variation of temperature and the flow of air. The variation of temperature will distort the mirror while the flow of air will affect the interferometric fringe. They both challenge high precision measurement. During testing process, we kept the testing room at a certain temperature. Then we enclosed the mirror and the interferometer in a small independent environment to decrease the flow of air. After measurement, we choose the result with higher reproducibility to start another CCOS run.

\section{RESULTS}

Polished surface: The relationship between grain size, polishing pressure, polishing velocity, material of polishing pad and resulting surface roughness is studied and the results are shown in Fig. 2.

Based on the experience from polishing experiments, we polish a $\mathrm{RB} \mathrm{SiC}$ sample and Fig. 3 shows the resulting surface roughness.

CCOS result: A $475 \mathrm{~mm}$ diameter sphere $\mathrm{RB} \mathrm{SiC}$ mirror was polished on our AOCMT (Aspheric Optical Compound Machining Tool). Figure 4 shows the converging process and Fig. 5 shows the initial surface error and final result.

\section{DISCUSSION}

Surface roughness: We can get follow information from Fig. 2:

- As a multiphase material (RB SiC contains $\mathrm{Si}, \alpha$ $\mathrm{SiC}$ and $\beta-\mathrm{SiC}$ ), the polishing process of $\mathrm{RB}-\mathrm{SiC}$ always accompany by "phase boundary destruction" and "local wear" phenomena. ("local wear" means during the polishing, the removal rate of $\mathrm{SiC}$ is more quickly than $\mathrm{Si}$, this is really abnormal considering the harder stiffness of $\mathrm{SiC}$. 


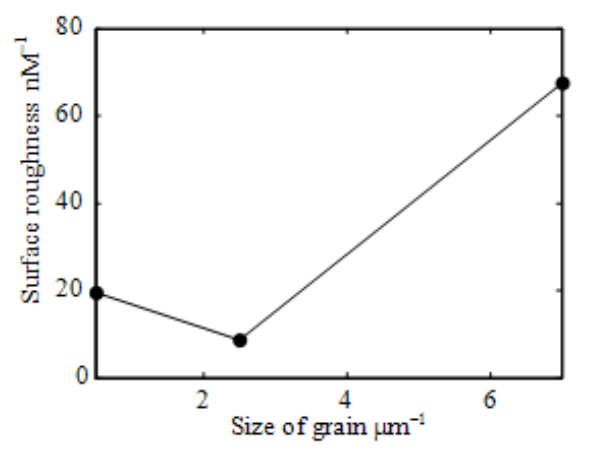

(a)

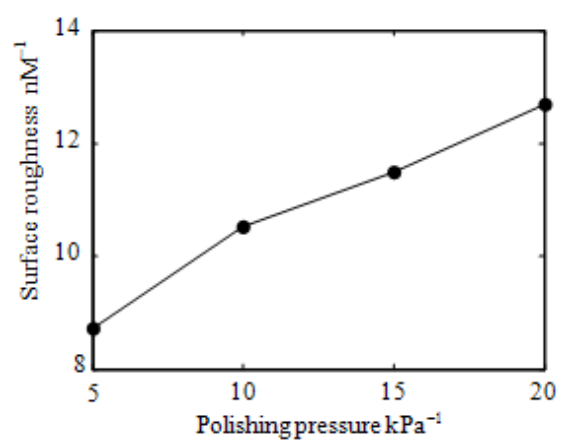

(b)

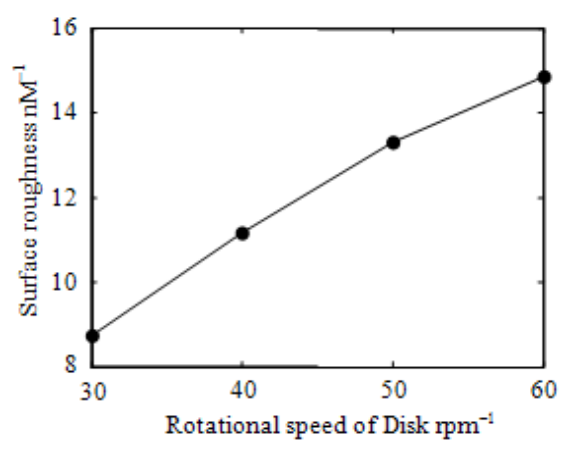

(c)

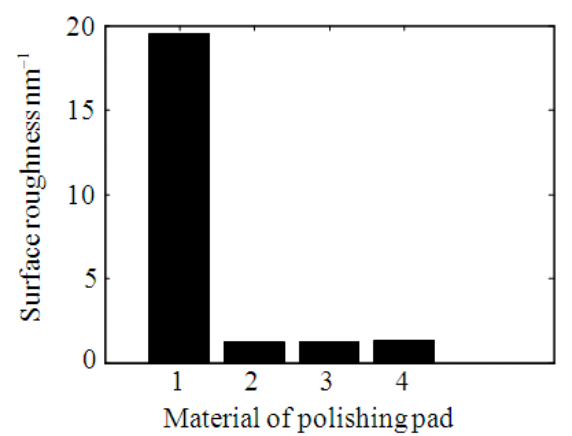

(d)

Fig. 2: Relationship of surface roughness between different polishing parameters "local wear" usually happens when polishing material with a fine polishing powder, such as W2.5 or W0.5 polishing powder). So Fig. 2a shows that the relationship between grain size and surface roughness is not linear. From the experiments we can know: When polishing $\mathrm{RB} \mathrm{SiC}$ with $\mathrm{W} 7$ diamond, surface roughness is bad because of "phase boundary destruction"; the surface roughness with W2.5 diamond is mainly decided by the balance height difference between $\mathrm{SiC}$ phase and $\mathrm{Si}$ phase; when polishing $\mathrm{RB} \mathrm{SiC}$ with $\mathrm{W} 0.5$ diamond, surface roughness is mainly decided by the material of polishing pad and polishing time as the "local wear" mainly affect the surface roughness

- Figure $2 \mathrm{~b}$ shows the relationship between surface roughness and polishing pressure. The figure tells us that higher polishing pressure leads to higher difference between $\mathrm{SiC}$ phase and Si phase, which results in worse surface roughness

- Figure $2 \mathrm{c}$ shows the influence of polishing velocity on surface roughness. When polishing velocity varies from 30-60 rpm, surface roughness becomes worse as velocity goes higher. This is mainly because as velocity goes faster, the distribution of polishing powder becomes more nonuniformly, which results in worse surface roughness

- Figure $2 \mathrm{~d}$ indicates how different polishing pad influence surface roughness. Several polishing pad like KSP 66A, 64\# pitch, 60\# pitch and 55\# pitch are supplied. We find that surface roughness of sample is in accordance to surface roughness of polishing pad. The surface roughness of KSP 66A is the worst, so it leads to serious "local wear" and the worst surface roughness. While pitch polishing pad all get surface roughness smaller than $2 \mathrm{~nm}$ rms due to their better surface roughness

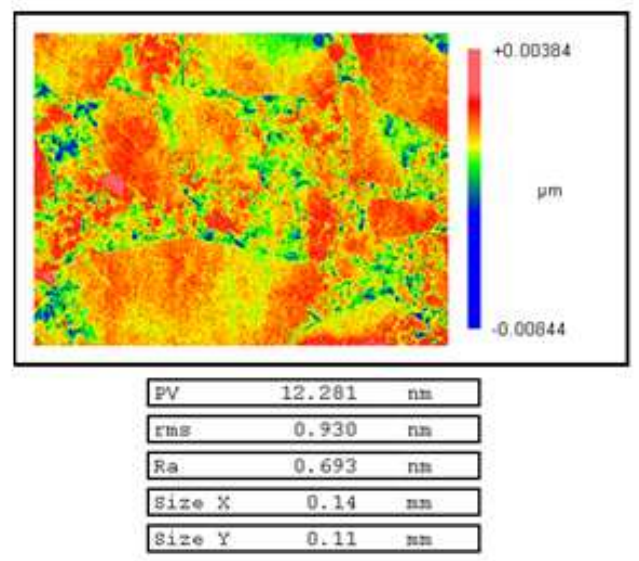

Fig. 3: Roughness of polished RB-SiC 


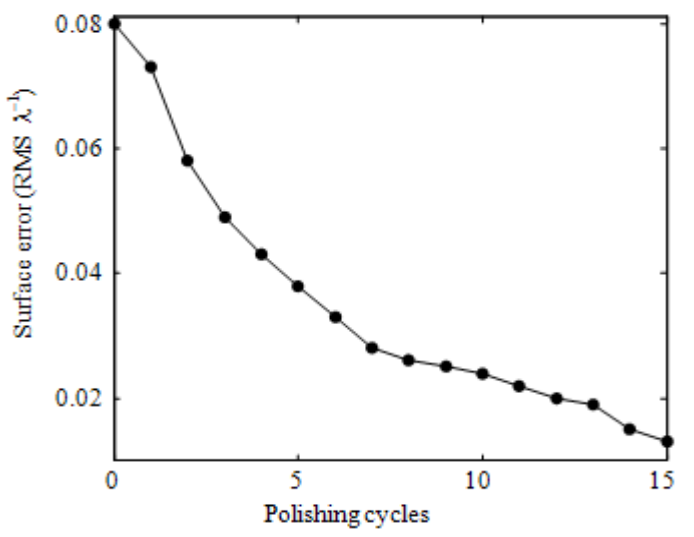

(a)

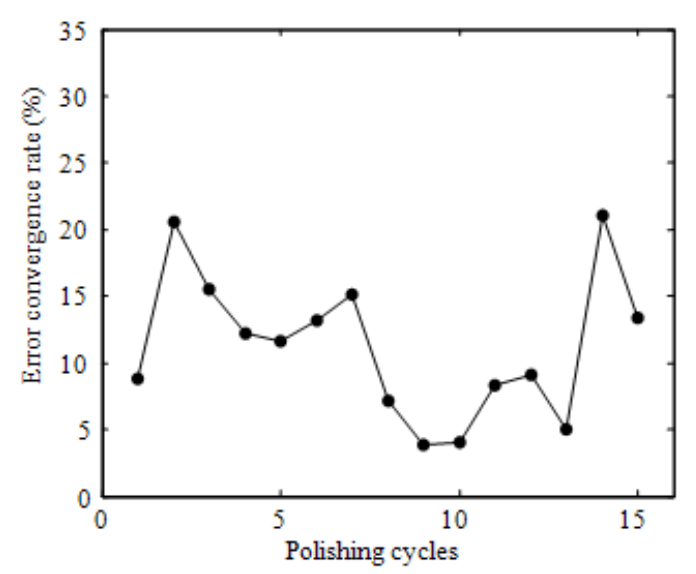

(b)

Fig. 4: Convergence of surface error

After all these experiment, we can know that polishing $\mathrm{RB}-\mathrm{SiC}$ with pitch, proper grain size, smaller polishing pressure and velocity could get better result. Based on these rules between polishing parameters and surface roughness, a RB-SiC sample is polished and a surface roughness of $0.930 \mathrm{~nm}(\mathrm{RMS})$ is obtained. The result is shown in Fig. 3.

CCOS process: Figure 4a shows the convergence curve of surface error (RMS) and Fig. 4b shows the error convergence rate of each cycle. From Fig. 4b, we can see that most cycles have a convergence rate between $5 \%$ and $15 \%$. The average improvement of the mirror per iteration is $11.2 \%$. The convergence rates vary from time to time, which means the polishing process is not very steady. There are a lot of reasons contributing to the instability, such as insufficient supply of polishing slurry, wear of polishing pad, nonuniform distribution of pressure between pad and workpiece, different surface error distribution of each cycle, We will proceed deeper research on the stability of CCOS and try to improve the convergence rate of it.

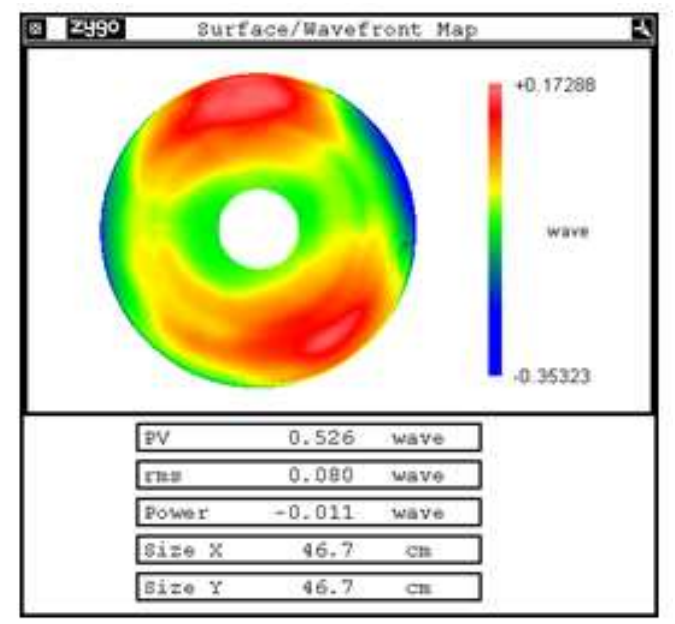

(a)

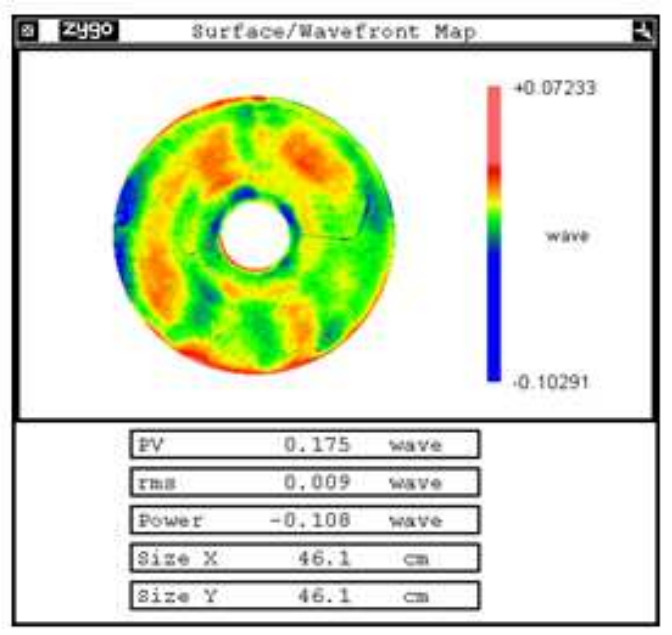

(b)

Fig. 5: Surface errors of the mirror (a) Before polishing (b) Final result

After polishing, surface error of the RB-SiC mirror reduced to $0.175 \lambda(\mathrm{PV}) / 0.009 \lambda$ (RMS) (97\% aperture) from $0.526 \lambda(\mathrm{PV}) / 0.080 \lambda$ (RMS). Figure 5 shows the surface error before and after polishing. From Fig. 5b, the $97 \%$ aperture of final surface of the mirror is better than $1 / 100 \lambda$ (RMS, $\lambda=632.8 \mathrm{~nm}$ ), representing a very good quality mirror.

\section{CONCLUSION}

The polishing parameters for $\mathrm{RB}-\mathrm{SiC}$ are optimized from polishing experiments. After a series experiment, we find ppolishing the $\mathrm{RB}-\mathrm{SiC}$ with pitch, proper grain size, smaller polishing pressure and velocity could get better result and a surface roughness better than $1 \mathrm{~nm}$ (RMS) is obtained. 
The basic theory and procedure of CCOS are introduced. Final surface figure better than $1 / 100 \lambda$ (RMS, $\lambda=632.8 \mathrm{~nm}$ ) proved the validity of CCOS method. Though the experiment mentioned in this study is based on sphere, but according to the sub-aperture process feature and strong flexibility of CCOS method, our next study will demonstrate the potentiality of this method for the manufacturing of highly accurate aspherical component.

\section{ACKNOWLEDGEMENT}

This study is supported by National Natural Science Foundation of China (Grand No. 50775216).

\section{REFERENCES}

Arneson, A., C. Alongi, R. Bernier, E. Boese and J. Daniel et al., 2010. Successful production of the Engineering Development Unit (EDU) primary mirror segment and flight unit tertiary mirror for JWST. Proc. SPIE, 7731: 77310I-1-77310I-11. DOI: $10.1117 / 12.858049$

Chen, X., P. Guo and J. Ren, 2010. Optimization of removal function in computer controlled optical surfacing. Proc. SPIE, 7655: 76551Y-1-76551Y-7. DOI: $10.1117 / 12.865694$
Kang, N., S. Li, Z. Zheng and Y. Dai, 2008. Experiment research on super-smooth polishing process of typical silicon carbide materials. China Mech. Eng., 21: 2528-2531+2535. http://www.cmemo.org.cn/EN/abstract/abstract836. shtml

Leal-Cabrera, I. and A. Cordero-Davila, 2009. Analytical and numerical classification of wear profiles produced with different-shape oscillating tools. Opt. Eng., 48: 103401-1-103401-9. DOI: $10.1117 / 1.3250168$

Peter, H. and M.R. Krodel, 2010. Designing optimized ultra-lightweighted mirror structures made of Cesic for space and ground based applications. Proc. SPIE, 7739: 77390J-1-77390J-15. DOI: $10.1117 / 12.856373$

Sus, M., R. Volkmer and P. Eisentrager, 2010. GREGOR M1 mirror and cell design: Effects of different mirror substrates on the telescope design. Proc. SPIE, 7739: 773911-1-77390J-13. DOI: 10.1117/12.857041

Zhang, A., Y. Chen, Z. Chen, H. Liu and J. Fang, 2010. Joining of silicon carbide ceramic for optical application by reaction bonded technology. Proc. SPIE, 7564: 746410-1-756410-5. DOI: $10.1117 / 12.865809$

Zheng, Y., X.-N. Li, W.-Y. Guo, Z.-C. Dong and B. Liang et al., 2010. Investigation of sub-aperture polish techniques for manufacturing astronomical mirror. Proc. SPIE, 7739: 77390E-1-77390E-8. DOI: $10.1117 / 12.856789$ 